\title{
Super-Oxidized Solution Nebulization for the Symptomatic Treatment of Airway Infections Including COVID-19 Cases Report
}

\author{
Dr. Roberto Carlos Rebollar González *, Dr. Edgar Torres López \\ Hospital Ángeles Lindavista. CDMX, México \\ Corresponding author: Dr. Roberto Carlos Rebollar González; rcrg_82@yahoo.com
}

Received 11 July 2020;

Accepted 21 July 2020;

Published 01 August 2020

\begin{abstract}
The coronavirus SARS CoV2 pandemic has had a great impact worldwide due to the disease contagious high degree and its range of clinical manifestations, from a mild to a severe condition that can end in death. The main transmission of the virus is by drops produced by coughing, sneezing, or talking that reach the host's airways. The initial location of the virus is in the nostrils and oropharynx during the first days of infection (viral phase); later it descends to the lower airway (pulmonary phase) where it can manifest itself in a more aggressive clinical condition. Until now, there is not treatment for this disease. This case series describes a proposal topical viricidal treatment in the respiratory tract by directly nebulized electrified super-oxidized solution (SOS). Thirty-two patients with epidemiological, clinical or imaging criteria for COVID-19 disease were included. The average age was 48.8 years, male sex $65 \%$, comorbidities $46 \%$ (diabetes mellitus, high blood pressure and obesity). Confirmatory test (SARS-CoV2 in oropharynx 6 and IgM 2) was obtained in 8 subjects. The average time of treatment started since onset of symptoms was $2.7(\mathrm{SD} \pm 1.8)$ days. No deaths or hospital admissions were reported. The average symptoms duration after treatment begun was $12.5(\mathrm{SD} \pm 2)$ days. Before treatment starting, average respiratory rate $(\mathrm{RR})$ was $22.6(\mathrm{SD} \pm 2.3)$ breaths per min (bpm) and average saturation of oxygen $(\mathrm{SO} 2)$ was $85(\mathrm{SD} \pm 6.5) \%$. Two days after starting treatment, a SO2 gradual increase was observed, as well as a RR gradual decrease. A $70 \%$ of subjects achieved $>93 \%$ SO2 from day 8, and 100\% reached RR <24 bpm at 4th day. From day 2, a decrease and amelioration in incidence and grade of discomfort was observed in practically all symptoms reaching less than $25 \%$ incidence of symptoms and on a scale of 2 to 4 at 10th day. The adverse effects (AE) associated with nebulization did not require treatment or suspend its application; less than $50 \%$ of cases reported headache, cough exacerbation, and dyspnea (more common AE). This case series proposes that nebulized SOS treatment improve a bad evolution (stops the deterioration of SO2 and RR) and improves the symptomatology of the COVID-19 disease.
\end{abstract}

Keywords: COVID-19 disease, SARS-CoV2, treatment, super-oxidized solutions, nebulization, average time symptoms

\section{Background}

The COVID-19 pandemic (SARS-CoV-2 coronavirus) started in China in December $2019^{[1]}$. In humans, several coronaviruses are known to cause respiratory infections that can range from the common cold to more serious illnesses such as Middle East respiratory syndrome (MERS) and severe acute respiratory syndrome (SARS) ${ }^{[2]}$. The most recently discovered coronavirus (SARS-CoV-2) causes coronavirus disease COVID-19 ${ }^{[3-7]}$. Most people (about 80\%) recover from COVID-19 disease without hospital treatment and will present a mild to moderate illness with symptoms similar to a cold $;^{[8]}$ the remaining $20 \%$ of the subjects may progress to critical situations such as pneumonia with the presence of oxygen desaturation after lung disease; of these ones, $5 \%$ of the subjects may progress to respiratory distress syndrome that may require management with invasive mechanical ventilation (critical stage of the illness); according to the age group, patients in this clinical state could die half or more of the cases ${ }^{[3,9]}$.
The disease spread by air when the virus is inhaled and established in the respiratory epithelium. The virus is contained in respiratory droplets of carriers, expelled by speaking, coughing, sneezing or spitting. It reach the host, directly to the mucosa in the mouth, nose, throat, trachea, lungs, ocular surface, or indirectly, through skin and fomites contaminated by these droplets autoinoculated in eyes, nose or mouth ${ }^{[8,10-16]}$. Other routes of spread have not been ruled out yet, however, the virus has been identified in faeces, peritoneal fluid, cerebrospinal fluid ${ }^{[10,15,17-19]}$. The viral loads in the pharynges is high during the first week, when the symptoms are moderate or prodromal, with a peak on day $4^{\text {th }[20-22]}$ this suggests active replication in the upper respiratory tract ${ }^{[8,14-16]}$ that have been proposed that the virus can enter through the mucous membranes, especially the nasal and laryngeal mucosa, then go down to the lungs through the respiratory tract ${ }^{[8,9,11,12]}$. The most common first symptoms of infection are cough and fever ${ }^{[23]}$. The virus can enter the peripheral blood from the lungs causing viremia, and the virus would subsequently attack targeted ACE2expressing organs such as lungs, heart, kidneys, and 
gastrointestinal tract ${ }^{[1,2,9]}$. Based on these assumptions, the clinical phase is divided into three: the viral phase, the acute phase (pneumonia phase) and the recovery phase ${ }^{[9]}$. If the immune function is effective in the acute phase (pneumonia phase) and there are no more diseases, the virus can be effectively suppressed and then the patient enter to the recovery phase ${ }^{[5,7]}$. If the patient is older, is in an immunodeficient state, or has other diseases such as hypertension and diabetes, has a high risk that the immune system cannot effectively control the virus in the acute phase (pneumonia phase), then the patient will progress to a severe or critical clinical condition $^{[7,9]}$.

In Mexico, the sentinel model of the Ministry of Health reported, at June, that $35 \%$ of the affected population had required hospitalization; $85.4 \%$ of those admitted correspond to those over 40 years of age; $56.5 \%$ of the male gender was affected. The estimated mortality, at the moment, was slightly higher than $11 \%$, the male gender occupied $67 \%$ of the total deaths and the predominant group was between $40-84$ years old ${ }^{\text {[24] }}$.

Infectious lung susceptibility arises from gas exchange architectural requirements and frequent inhalation of infectious agents. To promote ventilation, $100 \mathrm{~m}^{2}$ surface of the lung are continuously external environment exposed to satisfy the gas diffusion demand into a thin capillary tissue. Every minute, 5 to 10 Lt of ambient air is vented, also included particles, droplets, and pathogens ${ }^{[25]}$. Unlike other surfaces such as the cutaneous (wrapped in impervious skin), the digestive (acidic light in the upper tract and thick layer adherent mucus in the lower gastrointestinal tract), the lung surface has a large protected environmental interface with only minimal defence barrier and few obstacles on its way to the alveoli. Despite this structural permeability, the lungs successfully defend against most infectious challenges through a variety of mechanisms, such as mucus layer, mucociliary mechanics, antibody and antimicrobial peptides, alveolar macrophages, and leukocyte recruitment promoted by the pulmonary epithelium ${ }^{[26]}$. When basal defences are surpassed, the pulmonary epithelium responds by increasing its direct antimicrobial capabilities and directing leukocyte recruitment from the circulation ${ }^{[27]}$. Just as the accessibility and large surface area of the lungs contribute to infectious susceptibility, these features also provide a unique opportunity for topical therapy in the aerosol respiratory form ${ }^{[25,28,29]}$. Various approaches have been studied so far on topical therapy. Some of these approaches, it has been possible to increase mucociliary clearance using saline solution ${ }^{[29]}$. These are postulated to have the potential to reduce the pathogen load within the lungs ${ }^{[25]}$. Nebulized alcohol ${ }^{[30]}$ and hydroxychloroquine therapies ${ }^{[31]}$ have been postulated, for example, without being practiced.

Aerosol is a fine particles suspension or liquid drops in a gas, which can diffuse independently of breathing behaviour, and allow diseases treatment of the upper and lower airways (which share their pathogenic mechanisms) and which frequently they occur simultaneously as "united airway disease" ${ }^{[28]}$. The efficacy of this method is related to the size of the dispersed substance, preferably less than $10 \mu \mathrm{m}$, which allows good penetration into the bronchial tree and causes a high local concentration of a substance ${ }^{[32]}$. The most important practical advantage of this method is its simplicity and very low probability of adverse events as observed in a meta-analysis study where safety data was reviewed in 24 trials: 13 trials $(1,363$ neonates, 703 treated with hypertonic saline) reported no adverse event, and 11 trials (2,360 infants, 1,265 treated with hypertonic saline) reported at least one adverse event, most of which were mild and resolved spontaneously ${ }^{[29]}$.
Antiseptics are products that destroy or inhibit the growth of microorganisms in or over living tissue (e.g., healthcare personnel, sinks, and surgical scrubs) ${ }^{[33]}$. Defined by the WHO, an antiseptic is a disinfecting substance that when applied to the surface destroys or inhibits the growth of microorganisms in living tissues without causing harmful effects. To this group of antiseptics correspond the electrolyzed super-oxidized solutions (SOS) ${ }^{[34-38]}$.

The outcome of an electrolysis process with an electric current through sodium chloride solution produce SOS. These has a broad-spectrum efficacy against viruses, bacteria, yeasts and bacterial spores ${ }^{[39-42]}$. Landa Solis et. al. established the viricidal effect of the solution with its activity in vivo and on surfaces since $2005^{[38]}$. The mechanism of action is by means of an oxidizing effect directed at exposed sulfhydryl groups, particularly double bonds, altering essential cellular components, including lipids, proteins and RNA, basically managing to destroy the lipoprotein wall, direct damage to nucleic acids, hypo osmolarity effect and oedema of the microorganism ${ }^{[38,39,41]}$. The CDC established tissue toxicity index of the SOS in 10, which is minimal, being slightly above the $0.9 \%$ physiological solution. The components of SOS have derivates of chlorine ( 80 PPM) and free radicals similar that our body produces at different times and by different systems (immune system when coming into contact with a pathogenic microorganism), these substances are degraded after oxidizing, without being toxic at the concentrations included in the SOS; after 5 minutes of exposition with the tissues, the SOS turns to water and oxygen ${ }^{[38,39]}$. The SOS (like Microdacyn ${ }^{\mathrm{TM}}$ ) are manufactured under FDA (US Food and Drug Administration) and ISO 13485: 2003 regulations. Within these standard, it has studies under US EPA OPPTS 870.1300 registration of the inhalation toxicity, where no toxicity is observed at low doses (LD50 >5000 mg/kg), which makes it a noble substance for the airway.

At the moment, the components of SOS have not shown significant adverse topical or systemic reactions and in the literature, they are only reported between $0-1 \%$ due to the selectivity of their effect against the various microorganisms and not against host cells ${ }^{[40]}$.

Currently, COVID-19 does not have a specific antiviral treatment, at least within the known antivirals, however, we do have an antiseptic (SOS) with a powerful viricidal and non-toxic effect. Viral inactivation begins at 15 seconds, after 30 seconds more than $3 \log 10$ of the viral concentration have been eliminated ${ }^{[41]}$. Therefore, we propose as a therapeutic alternative, to nebulize SOS in the airway, to influence the respiratory epithelium (from the nasopharynx to the lung) in the viral phase, in order to decrease the viral load at the beginning of the disease ${ }^{[8,15,16]}$, hold in the process of the pulmonary phase and restrain the evolution to the severe phase and serious complications ${ }^{[2,4,9]}$. Decreasing the viral load can change the natural history of the disease.

\section{Material and Methods}

The study is a case series where a $5 \mathrm{ml}$ nebulized SOS therapy was applied, for $15 \mathrm{~min}$ three to four times a day for 7 days. The subjects were admitted to the protocolo almost consecutively, who attended a medical evaluation with a high suspicion of suffering from SARS-CoV2 infection, in the COVID-19 pandemic, in a private outpatient medical clinics at northern area of Mexico City and Ecatepec, State of Mexico in May and June 2020. Subjects with mild or moderate clinical condition, managed on an outpatient basis. Some subjects, despite having severe clinical status criteria for hospitalization, decided to continue their management at home. Inclusion criteria were adults over 18 years of age, both sexes, 
women of childbearing age (18 to 40 years), not pregnant, with suspected COVID-19 disease who had met any of the following sets of criteria: An epidemiological criterion and two clinician criterions; three clinical criterions; a clinical criterion with an evident image criterion. The documented epidemiological criteria were to have started with symptoms within 14 days of having contact with: a) verified infected subject; subject (s) who have had fever and/or respiratory symptoms COVID-19 or suspected COVID-19 or high risk; and, subject (s) who had fever and/or respiratory symptoms from a place such as home, office, classroom. The clinical criteria (COVID-19 respiratory symptoms) were: dry or productive cough, dyspnoea (feeling short of breath), anosmia (not perceiving odours), ageusia (not perceiving flavours), stuffy or runny nose, sore throat, headache, fever (home temperature $>38.2^{\circ} \mathrm{C}$ ), weakness or fatigue, chills, muscle and/or joint pain, digestive symptoms (diarrhoea, nausea, vomiting), as well as data on physical examination (findings in clinical review area) of respiratory rate $(\mathrm{RR})>22$ breaths per minute $(\mathrm{bpm})$, temperature $>38.2^{\circ} \mathrm{C}$, heart rate $(\mathrm{HR})>100$ beats per minute, pulse oximetry with oxygen saturation $<93 \%$ to ambient air, positive data in lung fields associated with secretions (thick rales, consolidation data), pleuropulmonary syndrome, shock state (clinical evidence of inadequate tissue perfusion), capillary refill $>3$ seconds after sustained pressure on the distal phalanx, mottled skin colour of the knee, low urinary output in 6 hours, altered mental state or disorder consciousness state (Glasgow <13), arterial hypotension, defined by a systolic pressure $<90 \mathrm{mmHg}$, mean arterial pressure $<65$ $\mathrm{mmHg}$ or a decrease of $40 \mathrm{mmHg}$ in arterial pressure baseline, serum lactate $>2 \mathrm{mmol} / \mathrm{L}$, exacerbation of cardiovascular or respiratory symptoms of underlying chronic diseases, glycemic decontrol (glycemia $>250 \mathrm{mg} / \mathrm{dl}$ ). The imaging criteria considered evident were: pulmonary ultrasound with Pattern B or more, bilateral, chest tele radiography with bilateral infiltrate, chest CT with high suspicion of COVID ${ }^{[42,43]}$. All patients who had identified clinical criteria in the evaluation that warranted hospitalization as at least two data from the qSOFA scale (quick Sequential Organ Failure Assessment): Respiratory rate >22 bpm; Systolic blood pressure <100mmHg; Glasgow Coma Scale $\leq 13$ ${ }^{[44]}$.Clinically, these patients present $\mathrm{RR}>22 \mathrm{bpm}$, with use of accessory muscles of respiration and clinical data of acute respiratory failure (diaphoresis, tachycardia, cyanosis, agitation) and deterioration of the state of consciousness, they were informed that they should receive hospital attention, however, there were patients who rejected this treatment modality, preferring to receive treatment at home, who were not excluded from the study. The following were considered as exclusion criteria: Subjects who had received hospital care. It was verified that the female subjects of gestational age (18-40 years) did not present a pregnancy, and those subjects or relatives who did not accept the management protocol. The elimination criteria were subjects who made the decision not to accept treatment, not to cooperate in the follow up, or to suspend the treatment, positive pregnancy test in women of gestational age in the follow up.

Within the clinical evaluation, the subjects were explained the management protocol to each patient and their responsible family member; Informed consent was obtained, evaluation (clinical review) was carried out to identify that the patient meets inclusion criteria, imaging studies such as pulmonary USG, chest $\mathrm{X}$-ray or chest CT scan were performed or requested, depending on the particular case, as well as (initial) laboratories and PCR for SARS-COV2. It was not an inclusion criterion to have a positive test for SARS-CoV2, since in some cases, for economic reasons not all subjects were able to do it. It was requested to obtain an ultrasonic nebulization equipment for treatment application, and a paper or electronic log (online form) was explained and provided to report the patient's symptoms; It was explained how to fill the daily records according to a visual analogue scale (VAS). It was indicated how to carry out the nebulization, preferably using a mask on top of the nebulization mask to provide a closed nebulization system, as well as nebulized in isolated and wellventilated room. Alarm data to be transferred to a COVID hospital in Mexico City was explained, as well as the manual to request services if required ${ }^{[23]}$. The follow-up method was explained and carried out by means of a daily text message and a telephone call 4 or 5 days, 10 to 12 days and three weeks after the first evaluation. The applied treatment protocol was paracetamol in all cases, azithromycin in some cases, standardized by the WHO. The main variables recorded daily by the subjects were SO2, RR, and symptoms on a VSC of 10 values. Adverse effects secondary to the nebulization application were also recorded, and explained when nebulization have to be suspended due to these adverse effects. Despite having requested the SARS-CoV2 PCR from all subjects, as well as laboratory and imaging studies, from the first evaluation, not all patients underwent the requested studies.

\section{Statistic Analysis}

Data analysis was performed using descriptive analysis of central distribution for quantitative parameters by averaging the variables of time, SO2 and RR in the days observed, together with the standard deviation (SD) to expose their variability. For qualitative data, they were described based on the incidence of recovery when $\mathrm{SO} 2$ was $>93 \%$ and $\mathrm{RR}<22 \mathrm{bpm}$, incidence of symptoms or AE, all converted into percentages. In addition, the average of the VAS of each symptom presented in the subjects on the days of observation was obtained.

\section{Results}

The case series consisted of a total of 32 subjects. Demographic characteristics were sex: 21 men (65\%), 11 women; average age of 48.8 years; comorbidities: 15 subjects $(46.8 \%)$, of whom 10 were diabetics, of these 10, 3 also had systemic arterial hypertension, 1 chronic smoker, and 4 had obesity (BMI $>30 \mathrm{~kg} / \mathrm{m} 2$ ). Nine of the subjects underwent PCR-SARS-CoV2 or immunoglobulin studies; 6 had positive SARS-COV2 PCR, one had a negative result; and 2 subjects had positive IgM. Thirteen subjects underwent pulmonary USG, identifying bilateral hepatization data in more than one location by hemithorax in 9 subjects (28\%), of which a subset of patients will be made later for analysis. The average time between the 1 st symptom appear and treatment onset was $2.7(\mathrm{SD}= \pm 1.8)$ days. The average duration of symptoms was $12.5(\mathrm{SD}= \pm 2)$ days.

The RR and SO2 were main indicators of the clinical status of the subjects. The baselines mean RR and $\mathrm{SO} 2$ before treatment beginning was $22.6 \mathrm{bpm}(\mathrm{SD} \pm 2.3)$ and $85 \%$ ( $\mathrm{SD} \pm 6.5)$. Two days after treatment beginning, a gradual increase in $\mathrm{SO} 2$ was observed, as well as a gradual decrease in RR (Figure 1). A SO2 >93\% (clinical recovery) was achieved in $70 \%$ of subjects on day 8 , without being $100 \%$. The RR below $22 \mathrm{bpm}$ was reached by $100 \%$ of the subjects at day 8 (Figure 2). It was observed, in patients ( 8 subjects) where was nebulized at the office, oxygenation improved during and after nebulization on average between 1-5\%. At the end of the 10 days of follow-up, a decrease in the average RR of $3 \mathrm{bpm}$ was observed and the final average $\mathrm{SO} 2$ was $93.5 \%$, achieving an average improvement of $8 \%$ points. At the end, 6 subjects (18.7\%) continued with supplemental oxygen requirements after the 10 days 
of registration. Patients with more intense symptoms or longer evolution time at the beginning of the treatment persisted with some residual symptoms without accompanying fever or ventilatory impairment such as asthenia, muscle pain or a slight irritative cough. No deaths or hospital admissions were reported.

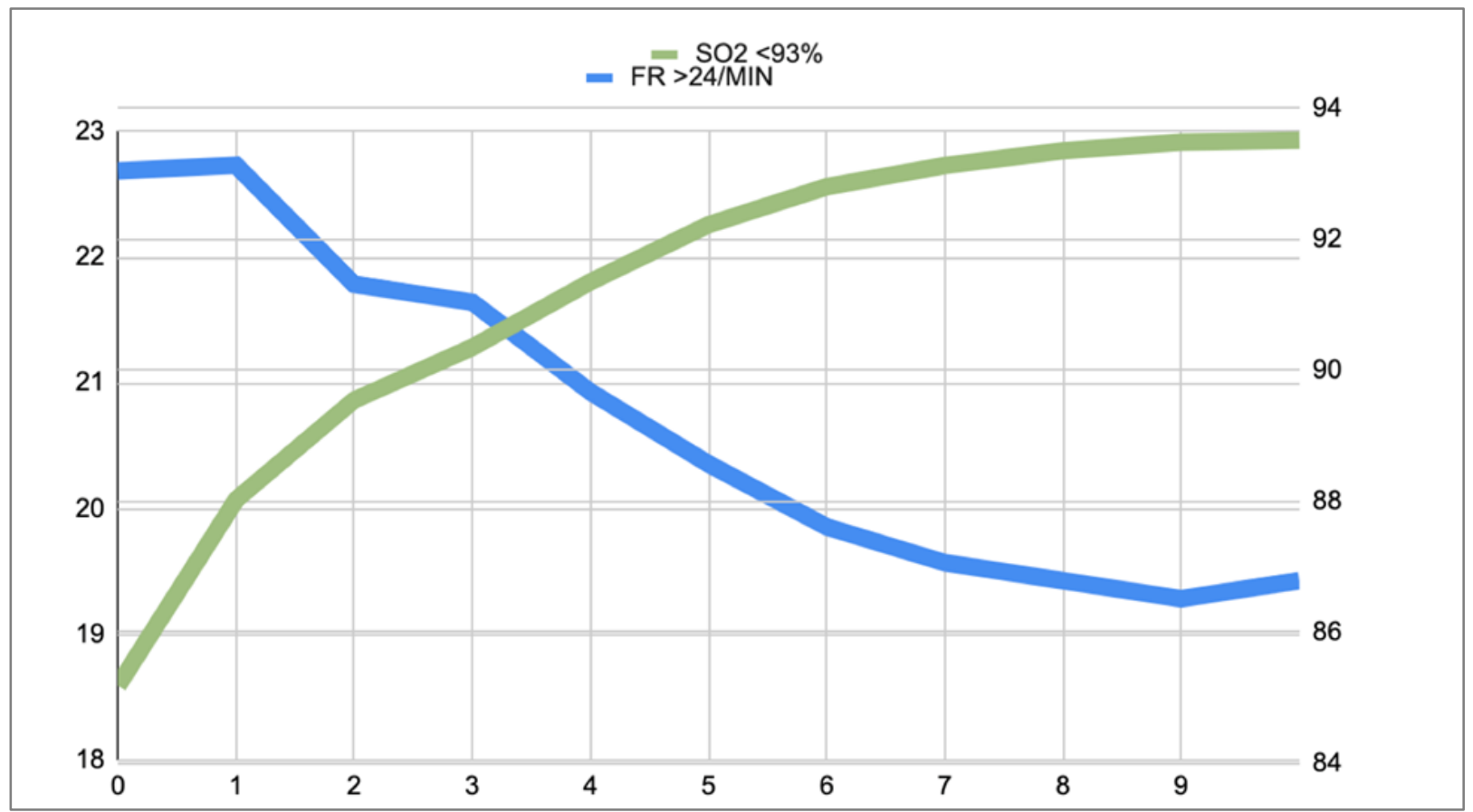

Figure 1. Evolution of Oxygen saturation (SO2) and respiratory rate from de beginning of treatment.

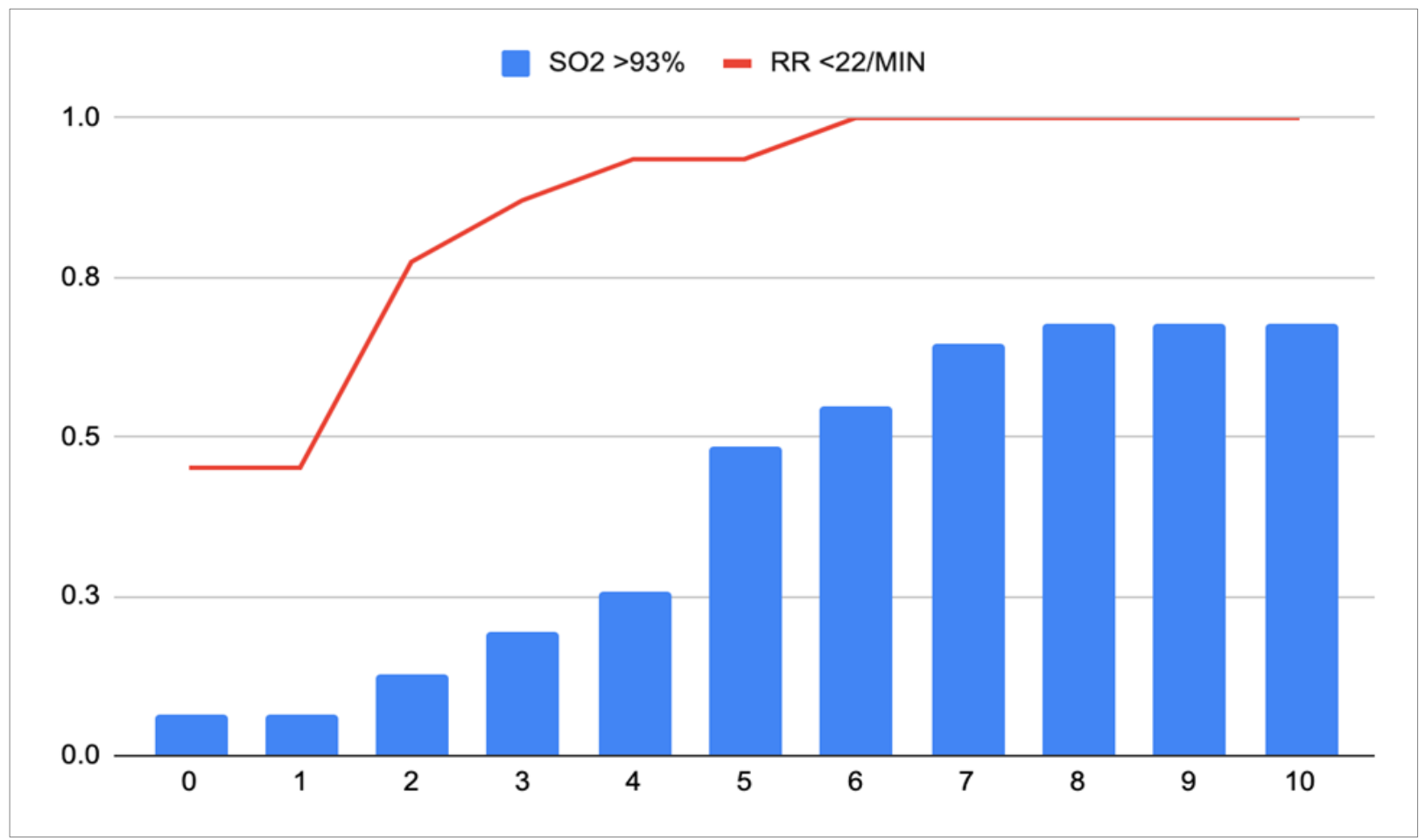

Figure 2. Evolution of the percentage of recovery of RR and SO2 after the SOS nebulized begun.

The average duration of symptomatic manifestations from the first day of symptom onset to the last symptom was 12.1 days, with the shortest being 3 days and the highest 21 days. In the cases of the subjects with the shortest duration with symptoms coincide with the subjects who started the treatment on the same day of the first symptom. It also coincides that the subjects who had the more severe clinical status and longest symptoms duration were the subjects who had the greatest time between onset of symptoms and the start of treatment.

The predominant symptom was weakness, followed by headache, chills, cough, muscle or joint pain, and fever at sixth place (Table 1), all of which were observed in more than $80 \%$ of the subjects. Unlike SO2 and RR, the decrease in the symptomatic presentation was observed from day 4 (Figure 3). Regarding the degree of symptom discomfort evaluated only if it was present, the symptoms with a score above 8 (very bothersome) on the 10 level VSC were dyspnoea, muscle and joint pain, and fever. Since day 2 , a linear decrease of the discomfort was observed in practically all the symptoms (Table 2), reaching a scale of 4 to 2 on day 10 (Figure 4). 


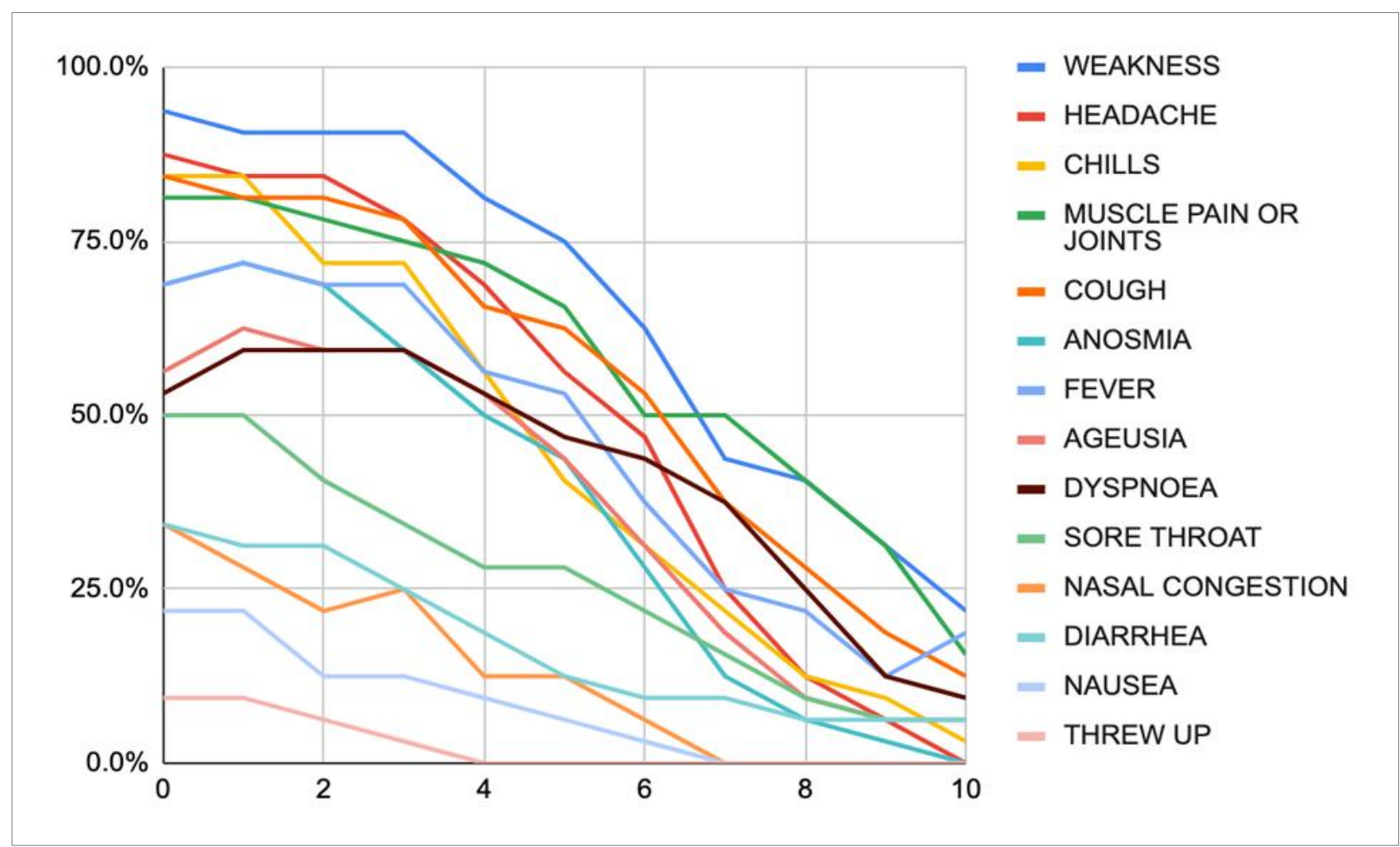

Figure 3. Incidence of symptoms within 10 days of nebulized SOS beginning. Day 0 is the (baseline) symptom before treatment started.

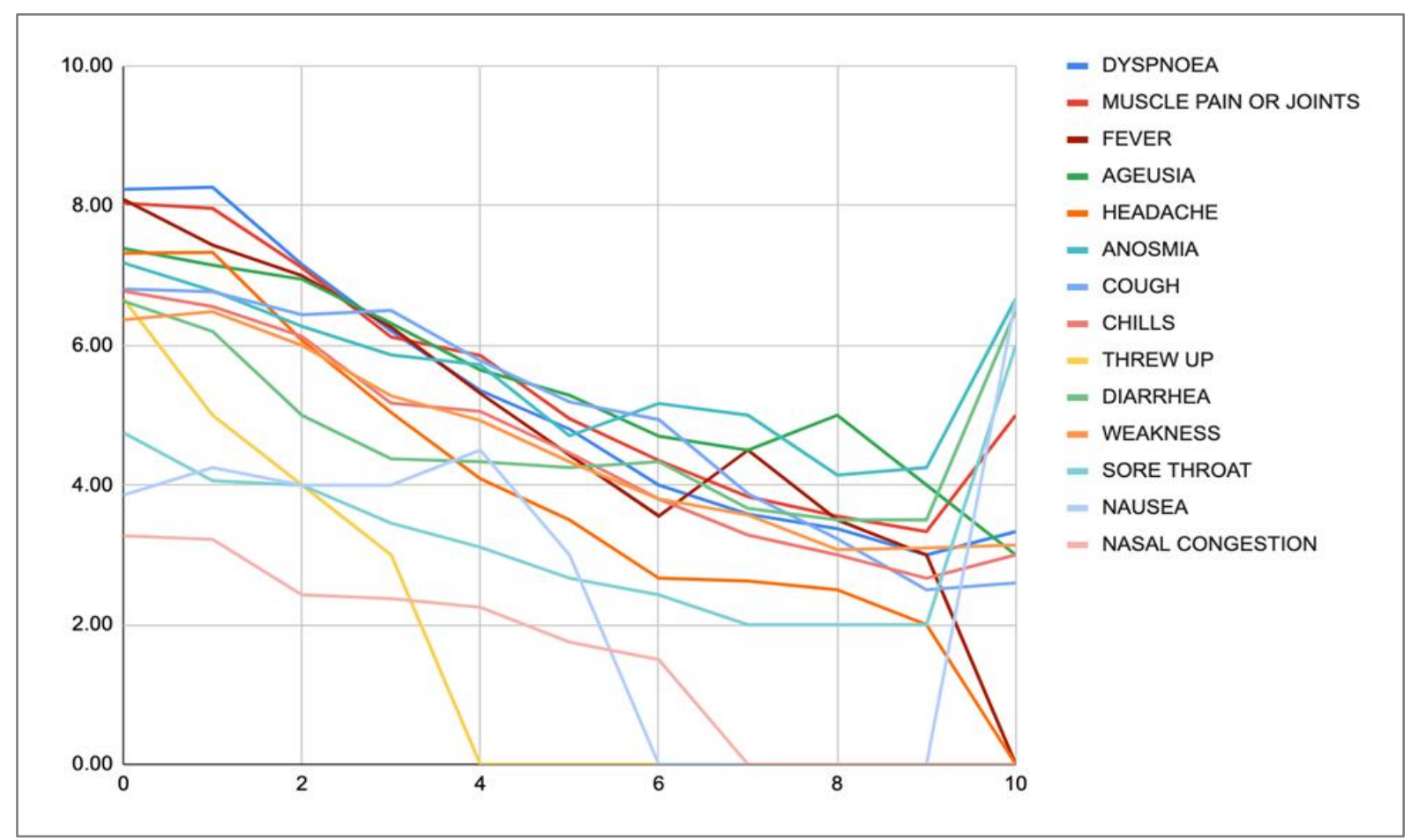

Figure 4. The evolution of VAS severity or discomfort of the symptom present in the $\mathbf{1 0}$ days following the start of the nebulized SOS. 


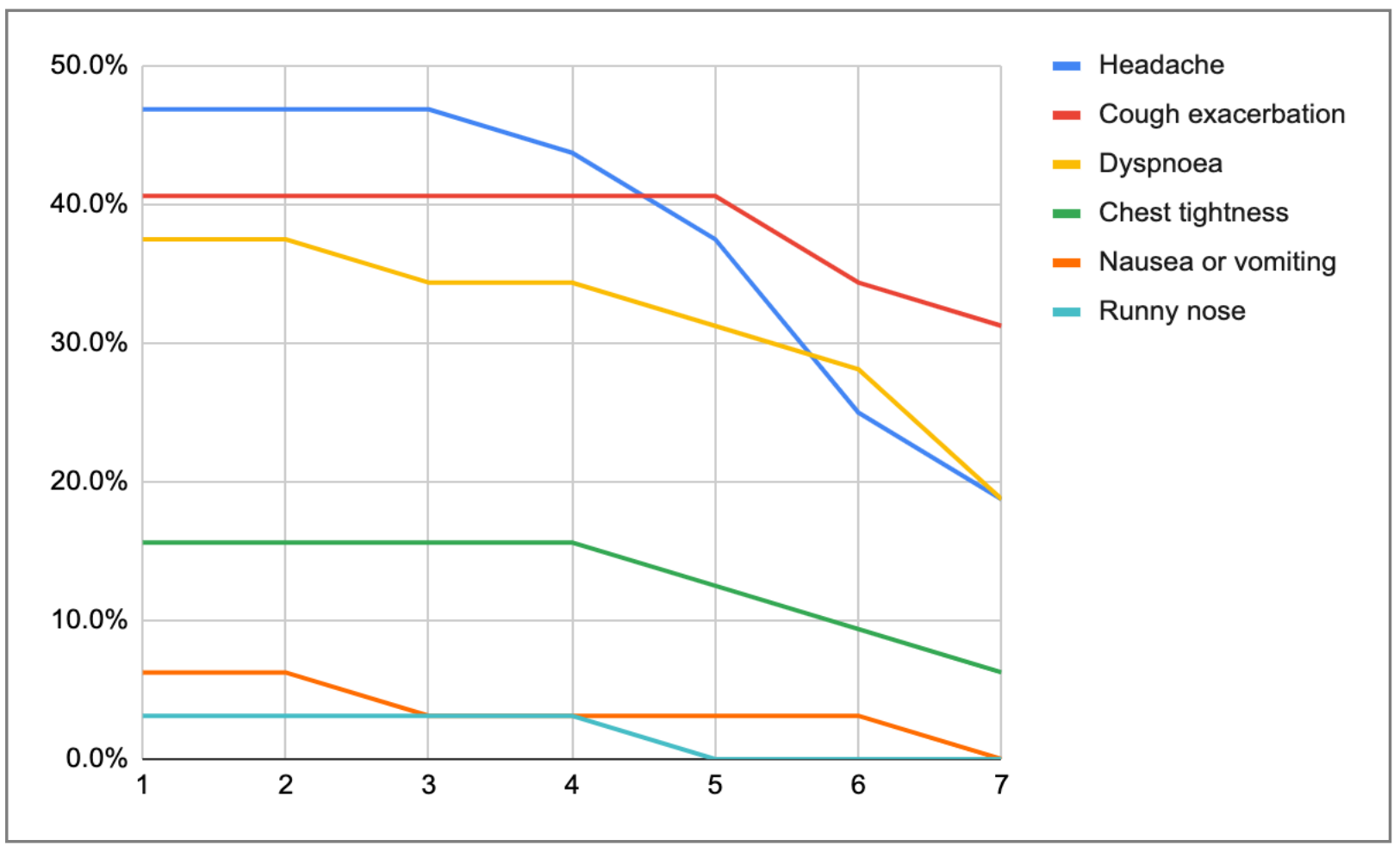

Figure 5. The incidence of AE associated with nebulized SOS within 7 days of its application

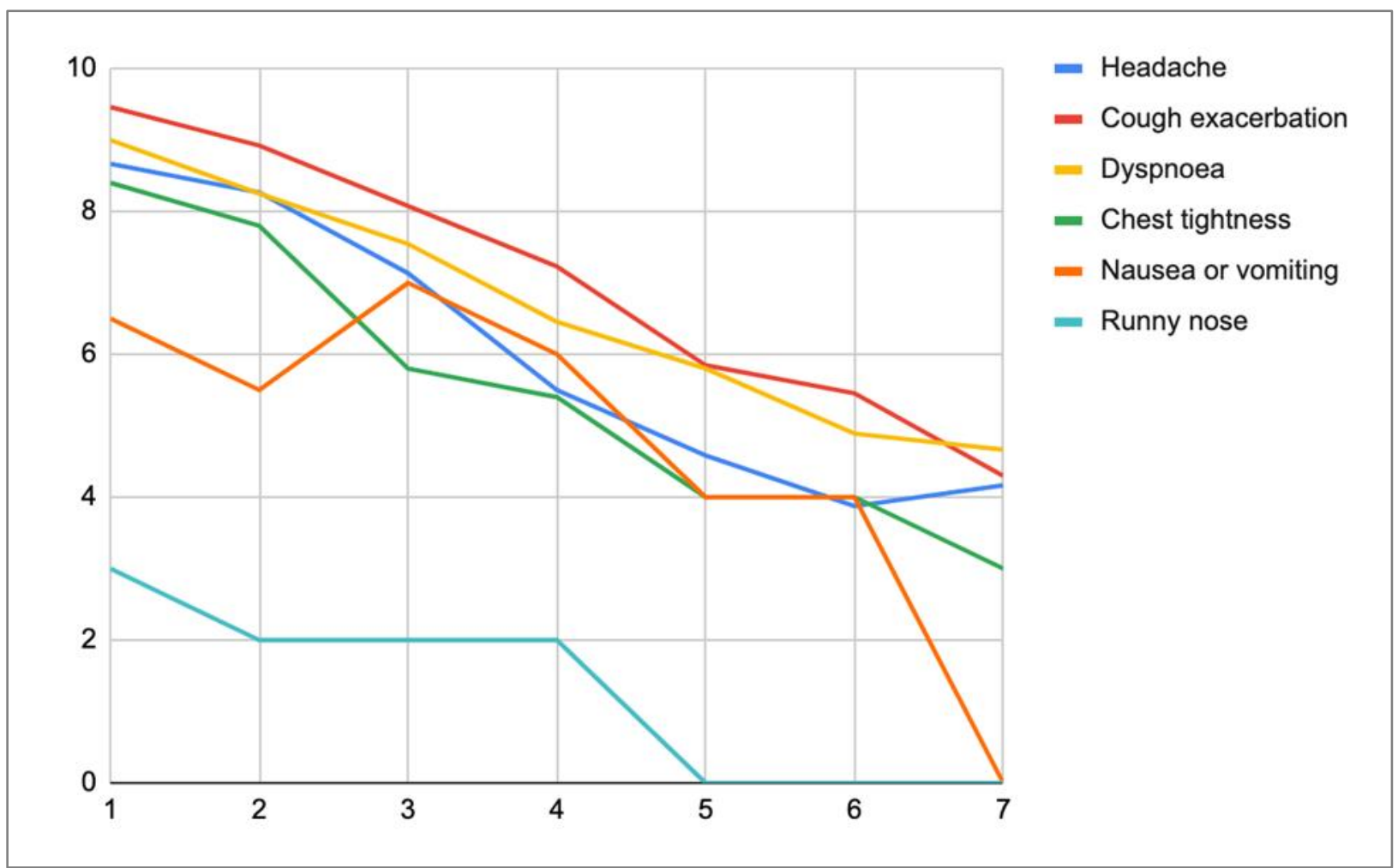

Figure 6. The degree of discomfort of adverse events on a visual analogue scale associated with SOS nebulized within 7 days of its application. 


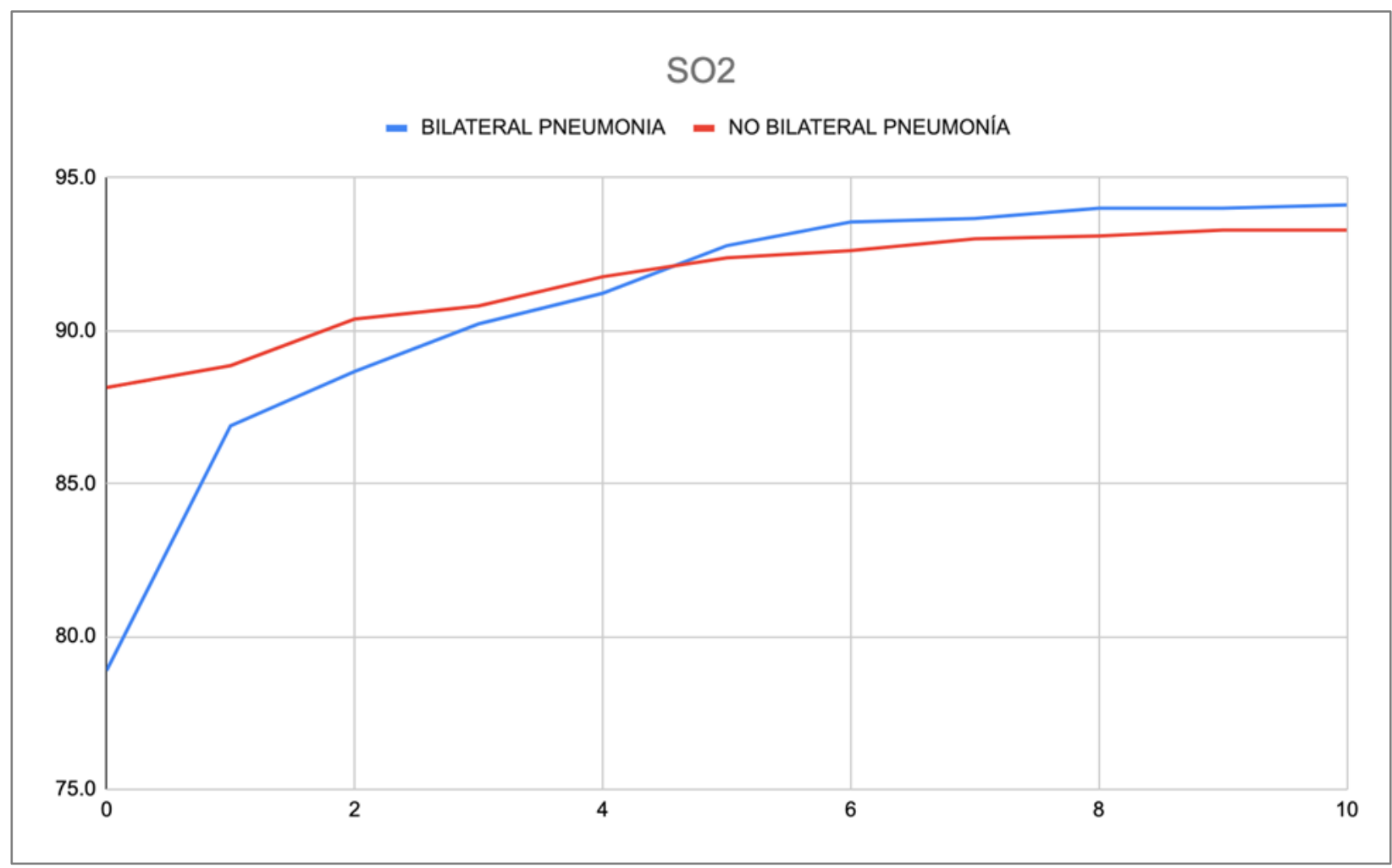

Figure 7. Difference in SO2 between subjects with bilateral pneumonia and the rest and its evolution from the beginning of treatment.

Table 1: Symptoms before the treatment, in order of presentation from most common to least common

\begin{tabular}{|l|c|}
\hline Symptom & INCIDENCE \\
\hline Weakness & $93.8 \%$ \\
\hline Headache & $87.5 \%$ \\
\hline Chills & $84.4 \%$ \\
\hline Muscle Pain Or Joints & $81.3 \%$ \\
\hline Cough & $81.3 \%$ \\
\hline Anosmia & $68.8 \%$ \\
\hline Fever & $68.8 \%$ \\
\hline Ageusia & $56.3 \%$ \\
\hline Dyspnoea & $53.1 \%$ \\
\hline Sore Throat & $50.0 \%$ \\
\hline Nasal Congestion & $34.4 \%$ \\
\hline Diarrhea & $34.4 \%$ \\
\hline Nausea & $21.9 \%$ \\
\hline Threw Up & $9.4 \%$ \\
\hline
\end{tabular}

Table 2: Decrease in AE compared from day 0 to day 7.

\begin{tabular}{|l|l|l|l|l|l|l|l|}
\hline Symptom & Days & 1 & 2 & 3 & 4 & 5 & 6 \\
\hline & 1 & $46.9 \%$ & $46.9 \%$ & $43.8 \%$ & $37.5 \%$ & $25.0 \%$ & $18.8 \%$ \\
\hline Headache & $43.8 \%$ & $40.6 \%$ & $40.6 \%$ & $40.6 \%$ & $40.6 \%$ & $34.4 \%$ & $31.3 \%$ \\
\hline Cough exacerbation & $40.6 \%$ & $37.5 \%$ & $34.4 \%$ & $34.4 \%$ & $31.3 \%$ & $28.1 \%$ & $18.8 \%$ \\
\hline Dyspnoea & $37.5 \%$ & $15.6 \%$ & $15.6 \%$ & $15.6 \%$ & $12.5 \%$ & $9.4 \%$ & $6.3 \%$ \\
\hline Chest tightness & $15.6 \%$ & $6.3 \%$ & $3.1 \%$ & $3.1 \%$ & $3.1 \%$ & $3.1 \%$ & $0.0 \%$ \\
\hline Nausea or vomiting & $6.3 \%$ & $3.1 \%$ & $3.1 \%$ & $3.1 \%$ & $0.0 \%$ & $0.0 \%$ & $0.0 \%$ \\
\hline Runny nose & $3.1 \%$ & & & & \\
\hline
\end{tabular}

Adverse effects (AE) associated with the nebulization were not cause of suspended o postponed the treatment and in no case was corrective treatment required to reconcile the $\mathrm{AE}$. The most observed $\mathrm{AE}$ was headache, followed by exacerbation of cough and dyspnoea. These three adverse effects occurred in less than $50 \%$ of the cases. The least frequent AE $\mathrm{s}$ were chest tightness (5 subjects), nausea or vomiting (2 subjects), and nasal congestion or runny nose only occurred in one subject in the first three days of treatment. No one present descent in SO2 during nebulization. No case reported dizziness, pain, irritation or inflammation of the throat.

\section{Discussion}

The COVID-19 pandemic affects the elderly and those with comorbidity with chronic degenerative diseases such as diabetes, 
systemic arterial hypertension and obesity with a worse prognosis [1]. In Mexico, the risk of hospitalization and death is higher than those reported worldwide, reaching $11 \%$ of deceased cases [24]. There is no treatment yet for COVID-19 disease. The SARS-CoV2 virus replicates in the nostrils and oropharynx during the first days (viral phase) and later in the lower airway (pulmonary phase) ${ }^{[9,44]}$. In this case series, a viricidal topical agent administered by nebulization on the epithelial surface of the respiratory tract was explored as an apparently effective and safe therapeutic option for the treatment of COVID-19 disease. This article opens the need to seriously explore its application in controlled clinical studies. The case series clearly identifies how well-established pneumonia patients managed to cope in less time than the disease usually takes. Positive changes in oxygen saturation were observed in patients who had frank deterioration due to illness after the start of the nebulization, since on the second day a progressive increase in oxygen saturation was observed, managing to restore their values above $93 \%$ oxygenation up to $70 \%$ of cases in less than 10 days. Thus, a favourable change was also observed in the respiratory rate and in the symptomatic limitation of the patients. In addition to these effects observed from the beginning of the proposed treatment, the authors perceived that patients started SOS nebulization as soon as possible, together with conventional treatment for COVID-19, achieve a faster improvement and less sequel and consequences than those patients who started treatment later, and with more advanced disease. It was also observed that even with a moderate to severe condition, patients achieved clinical improvement on average 4 days after treatment, progressively improving respiratory rate and oxygen saturation, as well as the incidence and perception of the intensity of the symptoms reported by patients. The proposed mechanism of action is to decrease the viral load in the respiratory epithelium. The direct viricidal effect of nebulized SOS reduces the amount of virus in the respiratory epithelium, at a burden that the natural immunity of the respiratory tract and especially of the lung can handle, with the consequent resolution of the pneumonic phase towards the recovery phase. The proposed treatment has several advantages:

a. Nebulization can be applied via an ultrasonic nebulizer, a portable device, which allows its application in outpatients. Some considerations should be taken, such as using a closed system and application in a wellventilated isolated room, at the time of the procedure to avoid the risk of transmission due to the formation of aerosols.

b. It does not interfere with any of the treatments and hygiene measures established by the WHO.

c. Probable universal application given its low or no toxicity and the formation of biodegradable residues, with manifestations of adverse effects already known from the effect of mists, but so far not serious that prevent their general application or warn their application for vulnerable populations. The reports of adverse reactions both from nebulization and from the application of this solution in other epithelia, in the international literature, is less than $1 \%$. The residues that form from SOS are found as components that the host defence cells themselves produce, such as hydrogen peroxide $\left(\mathrm{H}_{2} \mathrm{O}_{2}\right)$, ozone $\left(\mathrm{O}_{3}\right)$, oxygen $\left(\mathrm{O}_{2}\right)$ and water $\left(\mathrm{H}_{2} \mathrm{O}\right)$, which is why this type of solutions have been used widely and for more than two decades in the management of wounds, in abdominal sepsis and in high disinfection for medical components.
With these study we observed that super-oxidation solution nebulized is a potentially effective and safe treatment for viral airway infections. Therefore, it is necessary, urgently, to carry out randomized, blinded, controlled studies to assess the effectiveness and efficiency of the proposed therapy.

\section{Ethical Considerations}

According to the Declaration of Helsinki, which literally says "Article 37. When in the patient care the proven interventions there are no other known interventions, they have been ineffective, The doctor, after requesting expert advice, with the informed consent of the patient or of an authorized legal representative, can afford to use unproven interventions. Yes, in their judgment, they give some hope of saving life, restoring health, or alleviating suffering.

Such interventions should be further investigated in order to assess their safety and efficacy. In all cases, this new information must be recorded and, when appropriate, made available to the public. "... An exploration of a known low-risk toxicity solution is made using an innovative application not previously described in the medical literature.

\section{Reference}

[1] Chen Y, Liu Q, Guo D. Emerging coronaviruses: Genome structure, replication, and pathogenesis. J Med Virol. 2020. doi:10.1002/jmv.25681

[2] Zhou F YTDR, al. et. Correction. Clinical course and risk factors for mortality of adult inpatients with COVID-19 in Wuhan, China: a retrospective cohort study. Lancet 2020; published online March 9. https://doi.org/10.1016/S0140-6736(20)30566-3-. Lancet. 2020. doi:https://doi.org/10.1016/S01406736(20)30638-3

[3] Gorbalenya AE, Baker SC, Baric RS, et al. The species Severe acute respiratory syndrome-related coronavirus: classifying 2019-nCoV and naming it SARS-CoV-2. Nat Microbiol. 2020. doi:10.1038/s41564-020-0695-Z

[4] Le YH, Nguyen KC, Coleman KK, et al. Virus detections among patients with severe acute respiratory illness, Northern Vietnam. PLoS One. 2020. doi:10.1371/journal.pone.0233117

[5] Guarner J. Three Emerging Coronaviruses in Two Decades. Am J Clin Pathol. 2020. doi:10.1093/ajcp/aqaa029

[6] Lippi G, Sanchis-Gomar F, Henry BM. Coronavirus disease 2019 (COVID-19): the portrait of a perfect storm. Ann Transl Med. 2020. doi:10.21037/atm.2020.03.157

[7] Lipsitch M, Cohen T, Cooper B, et al. Transmission dynamics and control of severe acute respiratory syndrome. Science (80- $\quad$ ). 2003. doi:10.1126/science.1086616

[8] Zou L, Ruan F, Huang M, et al. SARS-CoV-2 viral load in upper respiratory specimens of infected patients. $N$ Engl J Med. 2020. doi:10.1056/NEJMc2001737

[9] Lin L, Lu L, Cao W, Li T. Hypothesis for potential pathogenesis of SARS-CoV-2 infection-a review of immune changes in patients with viral pneumonia. Emerg Microbes Infect. 2020;9(1):727-732. doi:10.1080/22221751.2020.1746199

[10] Sun J, Xiao J, Sun R, et al. Prolonged Persistence of SARS-CoV-2 RNA in Body Fluids. Emerg Infect Dis. 2020. doi:10.3201/eid2608.201097 
[11] Gaunt ER, Hardie A, Claas ECJ, Simmonds P, Templeton KE. Epidemiology and clinical presentations of the four human coronaviruses 229E, HKU1, NL63, and OC43 detected over 3 years using a novel multiplex real-time PCR method. J Clin Microbiol. 2010. doi:10.1128/JCM.00636-10

[12] Charlton CL, Babady E, Ginocchio CC, et al. Practical guidance for clinical microbiology laboratories: Viruses causing acute respiratory tract infections. Clin Microbiol Rev. 2019. doi:10.1128/CMR.00042-18

[13] Loeffelholz MJ, Tang YW. Laboratory diagnosis of emerging human coronavirus infections-the state of the art. Emerg Microbes Infect. 2020. doi:10.1080/22221751.2020.1745095

[14] Petruzzi G, De Virgilio A, Pichi B, et al. COVID-19: Nasal and oropharyngeal swab. Head Neck. 2020. doi:10.1002/hed.26212

[15] To KKW, Tsang OTY, Leung WS, et al. Temporal profiles of viral load in posterior oropharyngeal saliva samples and serum antibody responses during infection by SARS-CoV-2: an observational cohort study. Lancet Infect Dis. 2020. doi:10.1016/S1473-3099(20)30196-1

[16] Yu X, Sun S, Shi Y, Wang H, Zhao R, Sheng J. SARSCoV-2 viral load in sputum correlates with risk of COVID-19 progression. Crit Care. 2020. doi:10.1186/s13054-020-02893-8

[17] CDC C. The Novel Coronavirus Pneumonia Emergency Response Epidemiology Team. The Epidemiological Characteristics of an Outbreak of 2019 Novel Coronavirus Disease (COVID-19).; 2020.

[18] Chang D, Mo G, Yuan X, et al. Time Kinetics of Viral Clearance and Resolution of Symptoms in Novel Coronavirus Infection. Am J Respir Crit Care Med. 2020. doi:10.1164/rccm.202003-0524LE

[19] Yang JR, Deng DT, Wu N, Yang B, Li HJ, Pan X Ben. Persistent viral RNA positivity during recovery period of a patient with SARS-CoV-2 infection. J Med Virol. 2020. doi:10.1002/jmv. 25940

[20] Jiang X, Luo M, Zou Z, Wang X, Chen C, Qiu J. Asymptomatic SARS-CoV-2 infected case with viral detection positive in stool but negative in nasopharyngeal samples lasts for 42 days. J Med Virol. 2020. doi:10.1002/jmv.25941

[21] Li J, Zhang L, Liu B, Song D. Case Report: Viral Shedding for 60 Days in a Woman with COVID-19. Am J Trop Med Hyg. 2020. doi:10.4269/ajtmh.20-0275

[22] Bialek S, Boundy E, Bowen V, et al. Severe outcomes among patients with coronavirus disease 2019 (COVID19) - United States, February 12-march 16, 2020. Morb Mortal Wkly Rep. 2020. doi:10.15585/mmwr.mm6912e2

[23] Institutes C coordinator of national health and highly specialized hospitals. COVID-19 Guidelines for Patient Care.; $2020 . \quad$ https://coronavirus.gob.mx/wpcontent/uploads/2020/04/Lineamiento_Clinico_COVID19_CCINSHAE_14022020.pdf.

[24] COVID-19 Tablero México - CONACYT - CentroGeo GeoInt DataLab. https://coronavirus.gob.mx/datos/\#DOView. Accessed June 19, 2020.

[25] Safdar A, Shelburne SA, Evans SE, Dickey BF. Inhaled therapeutics for prevention and treatment of pneumonia. Expert Opin Drug Saf. 2009;8(4):435-449. doi: $10.1517 / 14740330903036083$
[26] Weers J. Inhaled antimicrobial therapy - barriers to effective treatment. Adv Drug Deliv Rev. 2015;85:24-43. doi:10.1016/j.addr.2014.08.013

[27] Elphick M, Von Hollen D, Pritchard JN, Nikander K, Hardaker LE, Hatley RH. Factors to consider when selecting a nebulizer for a new inhaled drug product development program. Expert Opin Drug Deliv. 2015;12(8):1375-1387. doi:10.1517/17425247.2015.1014339

[28] Yeo LY, Friend JR, McIntosh MP, Meeusen EN, Morton DA. Ultrasonic nebulization platforms for pulmonary drug delivery. Expert Opin Drug Deliv. 2010;7(6):663679. doi:10.1517/17425247.2010.485608

[29] House SA, Gadomski AM, Ralston SL. Evaluating the Placebo Status of Nebulized Normal Saline in Patients with Acute Viral Bronchiolitis: A Systematic Review and Meta-analysis. JAMA Pediatr. 2020;174(3):250-259. doi:10.1001/jamapediatrics.2019.5195

[30] Shintake T. Possibility of Disinfection of SARS-CoV-2 (COVID-19) in Human Respiratory Tract by Controlled Ethanol Vapor Inhalation. 2020;2:1-12. http://arxiv.org/abs/2003.12444.

[31] Klimke A, Hefner G, Will B, Voss U. Hydroxychloroquine as an aerosol might markedly reduce and even prevent severe clinical symptoms after SARS-CoV-2 infection. Med Hypotheses. 2020. doi:10.1016/j.mehy.2020.109783

[32] Karolewicz B, Nartowski K, Pluta J, Gorniak A. Physicochemical characterization and dissolution studies of acyclovir solid dispersions with Pluronic F127 prepared by the kneading method. Acta Pharm. 2016;66(1):119-128. doi:10.1515/acph-2016-0008

[33] Mcdonnell G, Russell AD, Operations L, Louis S. Antiseptics and Disinfectants: Activity, Action, and Resistance. Vol 12.; 1999.

[34] Organización Mundial de la Salud. Formulario Modelo de la OMS 2004. Formul Model la OMS 2004. 2004.

[35] You HS, Fadriquela A, Sajo MEJ, et al. Wound healing effect of slightly acidic electrolyzed water on cutaneous wounds in hairless mice via immune-redox modulation. Biol Pharm Bull. 2017. doi:10.1248/bpb.b17-00219

[36] Yahagi N, Kono M, Kitahara M, et al. Effect of electrolyzed water on wound healing. Artif Organs. 2000. doi:10.1046/j.1525-1594.2000.06557-3.x

[37] Rutala WA, Weber DJ. New Disinfection and Sterilization Methods. Vol 7.

[38] Landa-Solis C, González-Espinosa D, Guzmán-Soriano B, et al. Microcyn ${ }^{\mathrm{TM}}$ : A novel super-oxidized water with neutral $\mathrm{pH}$ and disinfectant activity. J Hosp Infect. 2005. doi:10.1016/j.jhin.2005.04.021

[39] Gutierrez A a. The science behind stable, super-oxidized water. Wounds. 2006

[40] Middleton AM, Chadwick M V., Sanderson JL, Gaya H. Comparison of a solution of super-oxidized water (Sterilox $\rho$ ) with glutaraldehyde for the disinfection of bronchoscopes, contaminated in vitro with Mycobacterium tuberculosis and Mycobacterium aviumintracellulare in sputum. $J$ Hosp Infect. 2000. doi:10.1053/jhin.2000.0772

[41] Gutiérrez AA, Thatcher E, Park R, Sciences OI. The Anti-Viral Efficacy of a New Super-Oxidized Solution. (1): 100 . 
[42] Greenhalgh T, Koh GCH, Car J. Covid-19: A remote assessment in primary care. BMJ. 2020;368:1-5. doi:10.1136/bmj.m1182

[43] World Health Organization (WHO). Coronavirus disease. World Heal Organ. 2020;2019(March):2633. doi:10.1001/jama.2020.2633

[44] Pandharipande PP, Shintani AK, Hagerman HE, et al. Derivation and validation of Spo2/Fio2 ratio to impute for Pao2/Fio2 ratio in the respiratory component of the Sequential Organ Failure Assessment score. Crit Care Med. 2009. doi:10.1097/CCM.0b013e31819cefa9

[45] Lauer SA, Grantz KH, Bi Q, et al. The incubation period of coronavirus disease 2019 (CoVID-19) from publicly reported confirmed cases: Estimation and application. Ann Intern Med. 2020;172(9):577-582. doi:10.7326/M20-0504 